\title{
Dissociation of Medial Frontal $\beta$-Bursts and Executive Control
}

\author{
Steven P. Errington, Geoffrey F. Woodman, and ${ }^{\circledR}$ Jeffrey D. Schall \\ Department of Psychology, Vanderbilt Vision Research Center, Center for Integrative and Cognitive Neuroscience, Vanderbilt Brain Institute, \\ Vanderbilt University, Nashville, Tennessee 37240
}

The neural mechanisms of executive and motor control concern both basic researchers and clinicians. In human studies, preparation and cancellation of movements are accompanied by changes in the $\beta$-frequency band $(15-29 \mathrm{~Hz})$ of electroencephalogram (EEG). Previous studies with human participants performing stop signal (countermanding) tasks have described reduced frequency of transient $\boldsymbol{\beta}$-bursts over sensorimotor cortical areas before movement initiation and increased $\boldsymbol{\beta}$-bursting over medial frontal areas with movement cancellation. This modulation has been interpreted as contributing to the trial-bytrial control of behavior. We performed identical analyses of EEG recorded over the frontal lobe of macaque monkeys (one male, one female) performing a saccade countermanding task. While we replicate the occurrence and modulation of $\beta$-bursts associated with initiation and cancellation of saccades, we found that $\beta$-bursts occur too infrequently to account for the observed stopping behavior. We also found $\beta$-bursts were more common after errors, but their incidence was unrelated to response time (RT) adaptation. These results demonstrate the homology of this EEG signature between humans and macaques but raise questions about the current interpretation of $\boldsymbol{\beta}$ band functional significance.

Key words: countermanding; EEG; error monitoring; response inhibition; stop signal; stopping

\section{Significance Statement}

The finding of increased $\beta$-bursting over medial frontal cortex with movement cancellation in humans is difficult to reconcile with the finding of modulation too late to contribute to movement cancellation in medial frontal cortex of macaque monkeys. To obtain comparable measurement scales, we recorded electroencephalogram (EEG) over medial frontal cortex of macaques performing a stop signal (countermanding) task. We replicated the occurrence and modulation of $\beta$-bursts associated with the cancellation of movements, but we found that $\beta$-bursts occur too infrequently to account for observed stopping behavior. Unfortunately, this finding raises doubts whether $\beta$-bursts can be a causal mechanism of response inhibition, which impacts future applications in devices such as brain-machine interfaces.

\section{Introduction}

Response inhibition and performance monitoring are executive control functions supporting goal-directed behavior. The countermanding (stop-signal) task provides insights into these functions (Verbruggen and Logan, 2008; Verbruggen et al., 2019). Recently, researchers have described a higher incidence of $\beta$-bursts in electroencephalogram (EEG) recorded over medial

\footnotetext{
Received Aug. 6, 2020; revised 0ct. 6, 2020; accepted 0ct. 8, 2020.

Author contributions: J.D.S. and G.F.W. designed research; J.D.S. and G.F.W. performed research; S.P.E. and J.D.S. analyzed data; S.P.E., G.F.W., and J.D.S. wrote the paper.

This work was supported by the National Eye Institute Grant R01-EY019882, P30-EY08126 and by Robin and Richard Patton through the E. Bronson Ingram Chair in Neuroscience. We thank B. Williams, R. Williams, M. Maddox, M. S. Schall, I. Haniff, S. Motorny, D. Richardson, L. Toy, and M. R. Feurtado for technical support. We also thank Dr. A. Bompas, Dr. K. A. Lowe, Dr. U. Rutishauser, Dr. A. Sajad, J. A. Westerberg, and Dr. T. Womelsdorf for useful conversations regarding the work.

The authors declare no competing financial interests.

Correspondence should be addressed to Jeffrey D. Schall at jeffrey.d.schall@vanderbilt.edu.

https://doi.org/10.1523/JNEUROSCI.2072-20.2020

Copyright $\odot 2020$ the authors
}

frontal cortex of humans during successful inhibition arising early enough to contribute to stopping the movement (Jana et al., 2020; Wessel, 2020). This result is difficult to reconcile with single-unit recordings within medial frontal cortex of macaques, which find neural signals modulating only after inhibition was achieved (Stuphorn et al., 2010) and most commonly after response inhibition errors (Stuphorn et al., 2000; Sajad et al., 2019).

The model of countermanding task performance as a race between GO and STOP processes (Logan and Cowan, 1984) offers clear criteria to attribute neural signals to movement initiation and inhibition when applied to electrophysiological (De Jong et al., 1995; Kok et al., 2004; Stahl and Gibbons, 2007; Godlove et al., 2011b; Reinhart et al., 2012; Swann et al., 2012; Wessel and Aron, 2015) and neurophysiological (Hanes et al., 1998; Paré and Hanes, 2003; Brown et al., 2008; Stuphorn et al., 2010; Schmidt et al., 2013; Brockett et al., 2020) measurements. To contribute to reactive response inhibition, a neural signal must be asserted before the STOP process finishes, and that 
signal must scale in probability of occurrence proportional to the probability of canceling a response after a stop signal is presented. The spiking rate of movement-related neurons in cortical (Hanes and Schall, 1995; Murthy et al., 2009; Mirabella et al., 2011) and subcortical (Paré and Hanes, 2003; Schmidt et al., 2013) motor circuits satisfy these criteria. When preparing responses, the activity of movement-related neurons accumulates to a fixed threshold. Variation in the rate of this accumulation produces variation in response latency. Responses are withheld when the accumulation of activity is interrupted after the stop signal. The reliability of this relationship has been evaluated by comparing the psychometric inhibition function of the probability of responding despite the stop signal with a neurometric function of the probability of neural activity exceeding a threshold (Brown et al., 2008). The neurometric functions derived from movement neurons in FEF mirror the inhibition function.

Meanwhile, the spiking rate of neurons in medial frontal areas do not (Scangos and Stuphorn, 2010; Stuphorn et al., 2010). Instead, such signals are thought to contribute to executive control. As performance of the countermanding task includes failures to cancel the response on about half of all stop signal trials. This high failure rate also affords analysis of neural signals of error processing, a feature of executive control which can interact with proactive control.

To elucidate their origin, we have been establishing systematically the homology of visual and cognitive EEG signals in macaque monkeys and humans and locating contributing cortical areas (Woodman, 2012). We have established the homology of the error-related negativity (ERN) between macaques (Godlove et al., 2011b) and humans (Reinhart et al., 2012) and identified a medial frontal source for the ERN (Sajad et al., 2019). Here, we seek to establish the homology of $\beta$-bursts sampled in EEG recorded over medial frontal cortex of macaques and humans. If so, then subsequent investigation with invasive approaches in monkeys can offer mechanistic insights into the relationship between $\beta$-bursts and response inhibition.

We found the incidence of $\beta$-bursts observed in the EEG of macaque monkeys paralleled observations in humans. However, the probability of $\beta$-bursts lagged far behind the probability of canceling responses. Unexpectedly, $\beta$-bursts were most common after errors of inhibition, but their incidence was unrelated to adaptation in response times (RTs). We conclude that these $\beta$-bursts in macaque monkeys are homologous to those in humans, supporting further invasive investigation. It appears that $\beta$-bursts may serve as a rough index of executive control processes, but they lack any causal efficacy on the stopping response and thus much theoretical or practical utility.

\section{Materials and Methods}

\section{Experimental model and subject details}

All procedures were in accordance with the National Institutes of Health Guidelines, the American Association for Laboratory Animal Care Guide for the Care and Use of Laboratory Animals and approved by the Vanderbilt Institutional Animal Care and Use Committee in accordance with the United States Department of Agriculture and Public Health Service policies. Data were collected from one male bonnet macaque (Eu, Macaca radiata, $8.8 \mathrm{~kg}$ ) and one female rhesus macaque (X, Macaca mulatta, $6.0 \mathrm{~kg}$ ) performing a saccade countermanding task (Hanes and Schall, 1995; Godlove et al., 2014). Both animals were on a $12 / 12 \mathrm{~h}$ light/dark cycle and all experimental procedures were conducted in the daytime. Each monkey received nutrient-rich, primate-specific food pellets twice a day. Fresh produce and other forms of environmental enrichment were given at least five times a week.

While human studies obtain data from more participants, for practical and regulatory reasons, data cannot be collected from as many monkeys. However, 25 years of investigating the stop signal task with macaque monkeys has shown that their performance matches in various nuanced details that of humans (Hanes and Schall, 1995) and the race model accounts for human and macaque performance equivalently (Boucher et al., 2007; Camalier et al., 2007). Investigations of this task by multiple nonhuman primate laboratories have found no differences beyond the incidental individual differences that are evident in human performance.

\section{Surgical procedures}

Surgical details have been described previously (Godlove et al., 2011a). Briefly, magnetic resonance images (MRIs) were acquired with a Philips Intera Achieva 3T scanner using SENSE Flex-S surface coils placed above or below the animal's head. T1-weighted gradient-echo structural images were obtained with a 3D turbo field echo anatomic sequence ( $\mathrm{TR}=8.729 \mathrm{~ms} ; 130$ slices, $0.70 \mathrm{~mm}$ thickness). These images were used to ensure Cilux recording chambers were placed in the correct area (Crist Instruments). Chambers were implanted normal to the cortex (monkey Eu: $17^{\circ}$; monkey X: $9^{\circ}$; relative to stereotaxic vertical) centered on midline: $30 \mathrm{~mm}$ (monkey Eu) and $28 \mathrm{~mm}$ (monkey X) anterior to the interaural line.

\section{Data collection protocol}

An identical daily recording protocol across monkeys and sessions was conducted. In each session, the monkey sat in an enclosed primate chair with their head restrained $45 \mathrm{~cm}$ from a CRT monitor (Dell P1130, background luminance of $0.10 \mathrm{~cd} / \mathrm{m}^{2}$ ). The monitor had a refresh rate of $70 \mathrm{~Hz}$, and the screen subtended $46^{\circ} \times 36^{\circ}$ of the visual angle. Eye position data were collected at $1 \mathrm{kHz}$ using an EyeLink 1000 infrared eyetracking system (SR Research). All data were streamed to a single data acquisition system (MAP, Plexon). Time stamps of trial events were recorded at $500 \mathrm{~Hz}$.

\section{Macaque electroencephalography}

The EEG was recorded from the cranial surface with an electrode located over medial frontal cortex. The electrode implants were constructed from Teflon-coated braided stainless-steel wire and solid-gold terminals. Implanted wires were cut to $8.5 \mathrm{~cm}$, the wire ends exposed, and gold Amphenol pins were crimped to both ends. One end of the wires was inserted into a plastic connector, whereas the gold pin on the other end was ground down until $1 \mathrm{~mm}$ of the pin remained. During aseptic surgery, a $\sim 1-\mathrm{mm}$ hole was drilled into the surface of the skull $(3-5 \mathrm{~mm}$ thick), allowing the terminal end of the electrode to be tightly inserted. The inserted gold pin was then covered with a small amount of acrylic cement. After the EEG electrode was implanted, the plastic connector was attached to exposed acrylic to allow access to the channels. Leads that were not embedded in the acrylic were covered by skin that was sutured back over the skull. This allowed for the EEG electrode to be minimally invasive once implanted. Unlike recordings from skull screws that extend to the dura mater through the skull, recordings from these electrodes approximate those used in human electrophysiological studies because the signals must propagate through the layers of brain, dura, and skull. Electrodes were referenced to linked ears using ear-clip electrodes (Electro-Cap International). The EEG from each electrode was amplified with a high-input impedance head stage (Plexon) and bandpass filtered between 0.7 and $170 \mathrm{~Hz}$. All data were streamed to a data acquisition system (MAP, Plexon).

\section{Saccade stop-signal (countermanding) task}

The saccade stop-signal task used in this study has been widely used previously (Hanes and Schall, 1995; Hanes and Carpenter, 1999; Cabel et al., 2000; Colonius et al., 2001; Kornylo et al., 2003; Morein-Zamir and Kingstone, 2006; Walton and Gandhi, 2006; Thakkar et al., 2011, 2015; Godlove and Schall, 2016; Wattiez et al., 2016; Verbruggen et al., 2019). 
Recently, a set of guidelines has been proposed for designing and analyzing the stop-signal task to allow for valid comparisons to be made across studies (Verbruggen et al., 2019). Our study followed all of the recommendations but two. These adjustments were necessary to obtain sufficient neural data and to address issues arising because monkeys gain so much more experience with the task parameters relative to human participants. First, $40 \%$ of trials in our study were stop trials, compared with the recommended $25 \%$. This higher value was used to achieve the necessary power to analyze neural data at the individual stop-signal level, but it did not introduce excessive slowing of responses (Emeric et al., 2007). Second, although we employed a staircase procedure, this stepped by one to three stop-signal delays (SSDs). We do this to prevent monkeys from anticipating the staircase (Nelson et al., 2010).

Briefly, trials were initiated when monkeys fixated a central point. Following a variable time period, the center of the fixation point was removed leaving an outline. At this point, a peripheral target was presented simultaneously on either the left or right hand of the screen. In this study, one target location was associated with a larger magnitude of fluid reward. The lower magnitude reward ranged from $0 \%$ to $50 \%$ of the higher magnitude reward amount. This incidence was adjusted to encourage the monkey to continue responding to both targets. The stimulus-response mapping of location-to-high reward changed across blocks of trials. Block length was adjusted to maintain performance at both targets, with the number of trials in each block determined by the number of correct trials performed. In most sessions, the block length was set at 10-30 correct trials. Erroneous responses led to repetitions of a target location, ensuring that monkeys did not neglect low-reward targets in favor of high-reward targets, a phenomenon demonstrated in previous implementations of asymmetrically rewarded tasks (Kawagoe et al., 1998).

On most of the trials, the monkey was required to make an eye movement to this target (no-stop trials). However, on a proportion of trials the center of the fixation point was re-illuminated (stop-signal trials); this stop signal appeared at a variable time after the target had appeared (SSD). An initial set of SSDs, separated by either 40 or $60 \mathrm{~ms}$, was selected for each recording session. The delay was then manipulated through an adaptive staircasing procedure in which stopping difficulty was based on performance. When a subject failed to inhibit a response, the SSD was decreased by a random step to increase the likelihood of success on the next stop trial. Similarly, when subjects were successful in their inhibition, the SSD was increased to reduce the likelihood of success on the next stop trial. This procedure was employed to ensure that subjects failed to inhibit action on $\sim 50 \%$ of all stop-signal trials. On nostop trials, the monkey was rewarded for making a saccade to the target. On stop-signal trials, the monkey was rewarded for withholding the saccade and maintaining fixation on the fixation spot. Following a correct response, an auditory tone was sounded $600 \mathrm{~ms}$ later, and followed by a high or low fluid reward, depending on the stimulus-response mapping.

\section{Experimental design and statistical analysis}

\section{Bayesian modeling of stop-signal performance}

As performance on the stop-signal task can be considered as the outcome of a race between a GO and STOP process, then a stop-signal reaction time (SSRT) can be calculated (Logan and Cowan, 1984). This value can be considered as the latency of the inhibitory process that interrupts movement preparation.

SSRT was estimated using a Bayesian parametric approach (Matzke et al., 2013a,b). Compared with classical methods of calculating SSRT (i.e., integration-weighted method; Logan and Cowan, 1984), this approach allows for a distribution of SSRT to be derived by using the distribution of reaction times on no-stop trials, and by considering reaction times on non-canceled trials as a censored no-stop RT distribution. Furthermore, this model also allows for the estimation of the probability of trigger failures for a given session (Matzke et al., 2017). Individual parameters were estimated for each session. The priors were bounded uniform distributions $\left[\mu_{G o}, \mu_{\text {Stop }}: U(0.001,1000) ; \sigma_{G o}, \sigma_{\text {Stop }}: U(1,500) \tau_{G o,}\right.$ $\tau_{\text {Stop }}: U(1,500)$; pTF: $\left.U(0,1)\right]$. The posterior distributions were estimated using Metropolis-within-Gibbs sampling and we ran multiple (3) chains. We ran the model for 5000 samples with a thinning of 5 .

\section{EEG processing and $\beta$-burst detection}

For each session, raw data were extracted from the electrode. This signal was then bandpass filtered between 15 and $29 \mathrm{~Hz}$. This signal was then epoched from -1000 to $2500 \mathrm{~ms}$ relative to multiple key events in a trial, including target onset, saccade, and stop-signal presentation. $\beta$-Burst detection was performed as previously described (Shin et al., 2017; Wessel, 2020). The description is adapted from therein. We then convolved the epoched signal for each trial with a complex Morlet wavelet of the form:

$$
\mathrm{w}(\mathrm{t}, \mathrm{f})=\mathrm{A} \exp \left(\frac{\mathrm{t}^{2}}{2 \sigma_{\mathrm{t}}^{2}}\right) \exp (2 \mathrm{i} \pi \mathrm{ft})
$$

with $\sigma=\frac{\mathrm{m}}{2 \pi \mathrm{f}}, \mathrm{A}=\frac{1}{\sigma_{t}} \sqrt{2 \pi}$, and $\mathrm{m}=7$ (cycles) for each of the 15 evenly spaced frequencies spanning the $\beta$ band $(15-29 \mathrm{~Hz})$. Time-frequency power estimates were extracted by calculating the squared magnitude of the complex wavelet-convolved data. Individual $\beta$-bursts were defined as local maxima in the trial-by-trial band time-frequency power matrix, for which the power exceeded a threshold of 6-times the median power of the entire time-frequency power matrix for the electrode. To compute the burst $\%$ across trials, we binary coded the time of the peak $\beta$-amplitude. A $\beta$-burst density function was generated by convolving the binary-coded array of $\beta$-burst activity with a Gaussian function of the form:

$$
\beta-\operatorname{bdf}(\mathrm{x})=\frac{1}{\sigma \sqrt{2 \pi}} \mathrm{e}^{-\frac{1}{2}\left(\frac{\mathrm{x}-\mu}{\sigma}\right)^{2}}
$$

where $\mu=00.0 \mathrm{~ms}$, and $\sigma=22.5 \mathrm{~ms}$. These values represent the time required for half a cycle at the median $\beta$-frequency.

\section{Behavioral comparisons between monkeys}

For each session, we extracted the mean response latencies on no-stop and non-canceled trials. After using the Bayesian approach described above, we extracted estimates of the SSRT mean and SD for each session. We also extracted estimates of the proportion of trigger failures for each session. Mean values between monkeys were compared using a one-way independent measure ANOVA. Greenhouse-Geisser corrections were applied when assumptions of sphericity were violated.

\section{Comparing $\beta$-bursts during stopping}

To examine how the $\beta$-bursts activity may vary dependent on trial type, the incidence of $\beta$-bursts observed during the stopping process were calculated for each session. The STOP process interval was defined as the time between SSD onset and SSRT. While stop trials by definition had a predefined SSD associated with them, no-stop trials were assigned an SSD value similar to that used in the most recent stop-trial. We compared this activity against a baseline period. This period was an equivalent interval of time ranging from $-200 \mathrm{~ms}$ before the target onset, to $-200 \mathrm{~ms}$ minus the mean SSRT on the given session. For each trial type and time period (baseline and stopping), we then calculated the proportion of trials in which at least one $\beta$-burst occurred. A two-way repeated measures ANOVA was conducted, with time window and trial type as factors, and the proportion of $\beta$-bursts as the dependent variable. This approach allowed us to determine whether $\beta$-bursts were more prevalent during particular trial types, and whether these events were clearly task-related activity. Post hoc tests were conducted if ANOVAs were statistically significant. To determine the time at which the incidence of $\beta$-bursts differentiated between non-canceled and canceled trials, we found the first time point at which the $95 \%$ confidence intervals (CIs) for the $\beta$-burst density functions no longer overlapped. GreenhouseGeisser corrections were applied when assumptions of sphericity were violated.

\section{Linking incidence of $\beta$-bursts to response inhibition}

To examine how neural function may reflect changes in stopping behavior, we looked at how the incidence of $\beta$-bursts varied with the probability of inhibiting a movement. This analysis was limited to SSDs with 15 
or more canceled trials. At each threshold, we subtracted the proportion of the $\beta$-bursts observed at a given SSD from the $\mathrm{p}$ (respond | stop-signal) at the same SSD. This difference between bursts and p (respond | stop-signal) was squared and values in the given session were summed, creating a sum of squared error between the two measures for each session and at each burst threshold. We then examined whether these values across sessions significantly differed from zero using a one-sample $t$ test at each threshold. We performed this analysis on both raw measures of $\beta$-burst proportions, and normalized measures, where incidences were relative to the maximum proportion of $\beta$-bursts observed. Findings were the same across both approaches.

\section{Linking $\beta$-burst incidence to error monitoring}

Error-related activity was examined by comparing the incidence of bursts on non-canceled trials at the middle most SSD to latency matched no-stop trials. Previous work from our lab has highlighted error-related spiking activity from this dataset became most prominent in the 100- to 300-ms period following an erroneous saccade (Sajad et al., 2019). As such, we calculated the incidence of $\beta$-bursts that occurred during this period. Across sessions we compared the incidence of bursts observed in error trials against those observed in trials where a saccade was correctly executed using a one-way repeated measures ANOVA. GreenhouseGeisser corrections were applied when assumptions of sphericity were violated.

\section{Linking $\beta$-bursts to post-error RT adaptation}

To examine how $\beta$-bursts may contribute to RT adaptations following errors or successful inhibition, we first quantified an index to capture the degree of slowing for each session. For post-error slowing, this was done by dividing the mean RT on no-stop trials following error trials by the mean RT on no-stop trials following no-stop trials for a given session. This value represents the proportional change in RT resultant from an error. We repeated this approach for post-stopping slowing, instead using the mean RT in no-stop trials following canceled trials as the numerator in this ratio in which the superscript identifies the current trial type, and the subscript identifies the preceding trial type:

$$
\begin{gathered}
\text { Post }- \text { error slowing index }=\frac{\mathrm{RT}_{\mathrm{Non}-\text { canceled }}^{\mathrm{No}-\text { top }}}{\mathrm{RT}_{\mathrm{No}-\text { stop }}^{\mathrm{No}}} ; \\
\text { Post }- \text { canceled slowing index }=\frac{\mathrm{RT}_{\text {Canceled }}^{\mathrm{No}-\text { stop }}}{\mathrm{RT}_{\mathrm{No}-\text { stop }}^{\text {No-stop }}} .
\end{gathered}
$$

For each monkey, we examined how the incidence of $\beta$-bursts observed in the error monitoring period of a given session varied with the given sessions post-error index. To determine the association between post-error $\beta$-burst activity and post-error slowing, we fit a generalized linear model. From this we extracted $R^{2}$ values and determined whether the observed slope was significant.

We compared the effects of previous trial outcome (trial $n-1$ ) on $\beta$-burst activity observed in the baseline of the following trial (trial $n$ ). We identified no-stop trials which immediately followed canceled, noncanceled, and no-stop trials and calculated the proportion of these trials in which $\beta$-bursts occurred in the -400 - to -200 -ms period before the target appearing. We then compared whether the proportion of $\beta$-bursts during this baseline period differed between different trial types using a one-way repeated measures ANOVA.

\section{Results}

We acquired 33,816 trials across 29 sessions from two macaques (Eu: 11,583; X: 22,233) performing the saccade stop-signal (countermanding) task (Fig. 1A). Both monkeys exhibited typical sensitivity to the stop-signal. Summary measures of performance are in Table 1. First, response latencies on non-canceled (error) trials were faster than those on no-stop trials (Fig. 1B, left). Second, the probability of failing to cancel and executing an erroneous saccade was greater at longer SSDs (Fig. $1 B$, right). These two observations validated the assumptions of the independent race model (Logan and Cowan, 1984), allowing us to estimate the SSRT, the time needed to cancel to partially prepared saccade.

Previous studies used SSRT for distinguishing whether neural signals can contribute directly to reactive control, so estimates of this duration must be accurate and precise. We calculated SSRT using a Bayesian parametric approach (Matzke et al., 2013a,b), which offers estimates of the SSRT for each session. The monkeys had indistinguishable mean SSRT (one-way independent measure ANOVA: $\left.F_{(1,27)}=0.108, p=0.745, \mathrm{BF}_{10}=0.367\right)$ and variance of SSRT (one-way independent measures ANOVA: $\left.F_{(1,27)}=0.819, p=0.819, \mathrm{BF}_{10}=0.360\right)$. This approach also quantified trigger failures when stopping was unsuccessful because the STOP process was not initialized. Trigger failures were significantly more common for monkey Eu relative to monkey $\mathrm{X}$ (one-way independent measures ANOVA: $F_{(1,27)}=18.458$, $\left.p<0.001, \mathrm{BF}_{10}=114.778\right)$.

\section{$\beta$-Bursts and response inhibition}

The monkeys' EEG was recorded with a lead placed on the cranial surface over the medial frontal cortex at location analogous to $\mathrm{FCz}$ in humans (Fig. 1C). At the individual trial level, $\beta$ band activity was characterized by obvious, burst-like events, rather than by steady changes in modulations (Fig. 1D). As observed in human studies (Jana et al., 2020; Wessel, 2020), the overall prevalence of these bursts was low during both baseline ( -400 to $-200 \mathrm{~ms}$ pretarget, $\sim 12.6 \pm 3.8 \%$ across all sessions) and taskrelevant ( $0-200 \mathrm{~ms}$ post-target, $\sim 16.1 \pm 5.2 \%$ across all sessions) periods.

Following previous studies (Jana et al., 2020; Wessel, 2020), to examine the relationship between $\beta$-burst activity and stopping behavior in the countermanding task, we first compared the prevalence of $\beta$-bursts across trial types (two-way repeated measures ANOVA with time window and trial type as factors, Greenhouse-Geisser corrected: $F_{(1.72,48.31)}=9.816, \quad p<0.001$, $\mathrm{BF}_{10}=53.763$; Fig. $2 A$; Table 2). We found no significant changes in the incidence of $\beta$-bursts in a baseline period and during the stop process on non-canceled trials (Holm post hoc test, adjusted $p=0.300$ ) or during an equivalent period of time when stopping would have occurred on no-stop trials (Holm post hoc test, adjusted $p>0.999)$. However, compared with a baseline period, $\beta$-bursts were significantly more common during the STOP process when a movement was successfully canceled (Holm post hoc test, adjusted $p=0.019$ ). Furthermore, $\beta$-bursts were significantly more common during the STOP process on canceled compared with non-canceled trials (post hoc test, adjusted $p<0.001$ ) but not compared with an equivalent period of time on no-stop trials (post hoc test, adjusted $p=0.057)$. This pattern of $\beta$-burst incidence replicates previous reports from human participants (Jana et al., 2020; Wessel, 2020).

Neurophysiological investigations quantify neural signals on a finer time scale using spike density functions (Hanes et al., 1998). To examine how changes in $\beta$-bursts occur over time, we derived $\beta$-burst density functions. First, we binary coded the time of the peak $\beta$-amplitude (Fig. $2 B$ ). A $\beta$-burst density function was determined by convolving this discretized array with a Gaussian function over time since the stop-signal (Fig. 2C). These plots reveal more information about the dynamics of $\beta$-burst production through trial time and across trial types. $\beta$-Burst frequency increases through the trial during saccade preparation. On no stop trials and non-canceled trials, $\beta$-burst 

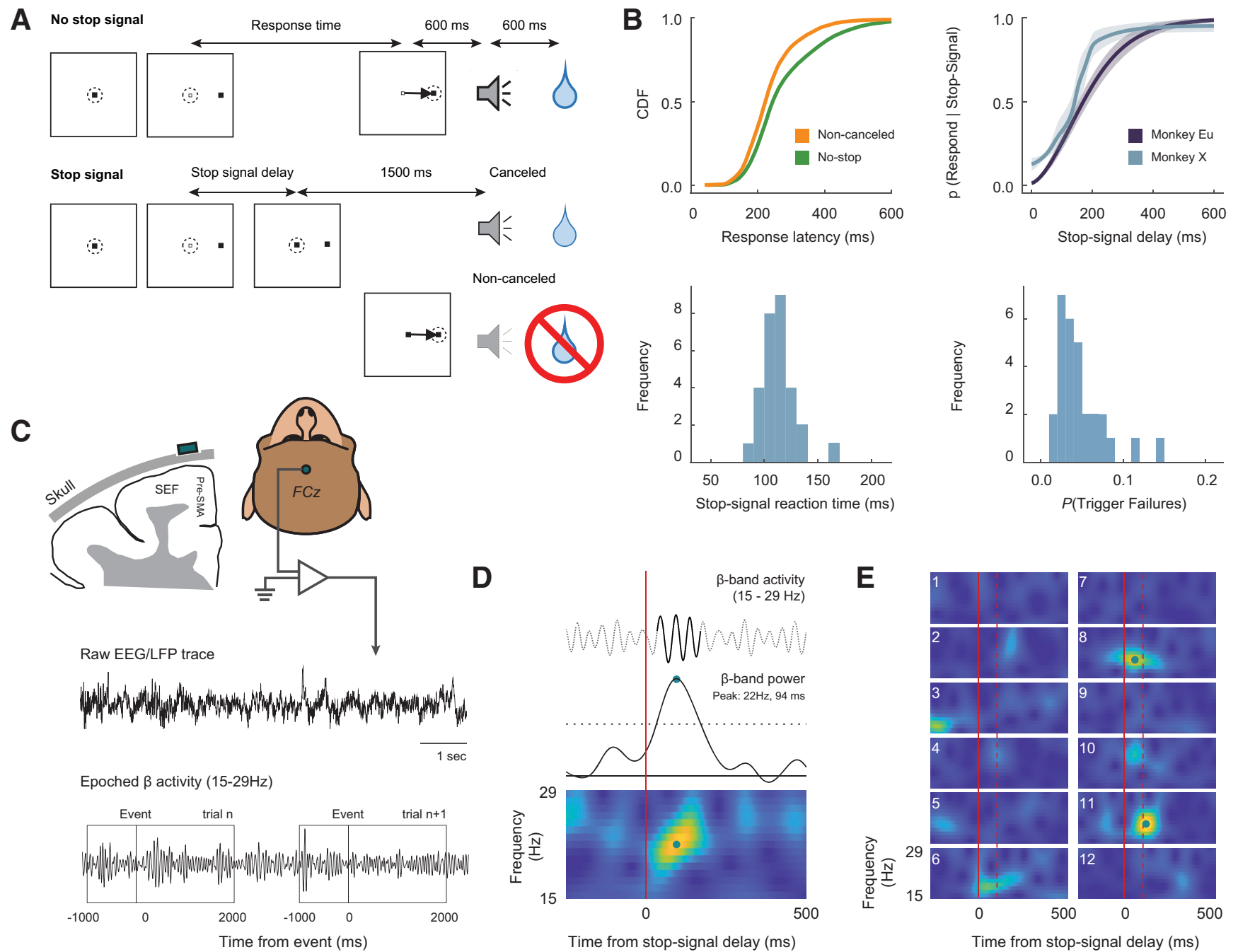

Figure 1. Experimental procedures. A, Saccade-countermanding task. Monkeys initiated trials by fixating on a central point. After a variable time, the center of the fixation point was extinguished. A peripheral target was presented simultaneously at one of two possible locations. On no-stop-signal trials monkeys were required to shift gaze to the target, whereupon after $600 \pm 0 \mathrm{~ms}$ a high-pitched auditory feedback tone was delivered, and 600 -ms later fluid reward was provided. $0 \mathrm{n}$ stop-signal trials ( $\sim 40 \%$ of trials), after the target appeared the center of the fixation point was re-illuminated after a variable SSD, which instructed the monkey to cancel the saccade in which case the same high-pitched tone was presented after a $1500 \pm 0$ ms hold time followed, after $600 \pm 0$ ms by fluid reward. SSD was adjusted such that monkeys successfully canceled the saccade in $\sim 50 \%$ of trials. In the remaining trials, monkeys made non-canceled errors which were followed after $600 \pm 0$ ms by a low-pitched tone, and no reward was delivered. Monkeys could not initiate trials earlier after errors. B, Countermanding behavior. Top left, Cumulative distribution function of response latencies on no-stop (green) and non-canceled (yellow) trials. Response latencies on non-canceled trials were faster than those on no-stop trials. Top right, Inhibition function plotting the probability of responding across SSDs. Weibull functions were fitted to data from each session. The mean of these Weibull functions across sessions and the corresponding $95 \% \mathrm{Cl}$ is plotted for each monkey (monkey Eu: purple; X: blue). Bottom left, Distribution of mean SSRTs across sessions. Bottom right, Distribution of the proportion of trigger failures across sessions. C, LFP processing. EEG was recorded with leads placed on the cranial surface over the medial frontal cortex at the location analogous to FCz in humans. The EEG lead was located over the supplementary eye field (SEF) and pre-supplementary motor area (pre-SMA). For each session, raw data were extracted. After bandpass filtering between 15 and $29 \mathrm{~Hz}$, this signal was epoched from -1000 to $2500 \mathrm{~ms}$ relative to target presentation, saccade initiation, and stop-signal presentation. $\boldsymbol{D}, \beta$-Burst processing. The epoched signal for each trial was convolved with a complex Morlet wavelet. Time-frequency power estimates were extracted by calculating the squared magnitude of the complex wavelet-convolved data. Individual $\beta$-bursts were defined as local maxima in the trial-by-trial band time-frequency power matrix, for which the power exceeded a threshold of six times the median power of the entire time-frequency power matrix for the electrode. An example burst is shown in the time-frequency plot at the bottom. $\boldsymbol{E}$, Examples of $\beta$ band time frequency in 12 randomly selected trials, aligned on SSD (solid red), with the corresponding SSRT (dashed red). These plots are indistinguishable from counterparts derived from human data.

Table 1. Stop-signal task performance (mean \pm SEM) for both monkeys across all sessions

\begin{tabular}{lcc}
\hline & Monkey Eu ( $n=12$ sessions) & Monkey X ( $n=17$ sessions) \\
\hline No-stop RT (ms) & $313.4 \pm 1.6$ & $263.0 \pm 1.0$ \\
Non-canceled RT (ms) & $259.4 \pm 2.0$ & $229.6 \pm 1.0$ \\
SSRT $_{\text {mean }}(\mathrm{ms})$ & $112.4 \pm 6.4$ & $114.3 \pm 1.8$ \\
SSRT $_{\text {std }}(\mathrm{ms})$ & $30.5 \pm 1.8$ & $30.9 \pm 1.0$ \\
$p$ (trigger failures) & $0.069 \pm 0.010$ & $0.032 \pm 0.003$ \\
\hline
\end{tabular}

frequency decreases following saccade initiation. The decrease in non-canceled trials begins earlier because the latency of non-canceled saccades is systematically less than that of no-stop trials. However, after noncancelled errors the incidence of $\beta$-bursts observed increases markedly. This will be characterized further below.

The incidence of $\beta$-bursts differentiated between correctly inhibited or incorrectly executed stop trials on average across sessions $132 \mathrm{~ms}$ after a stop-signal appeared. However, unlike the 


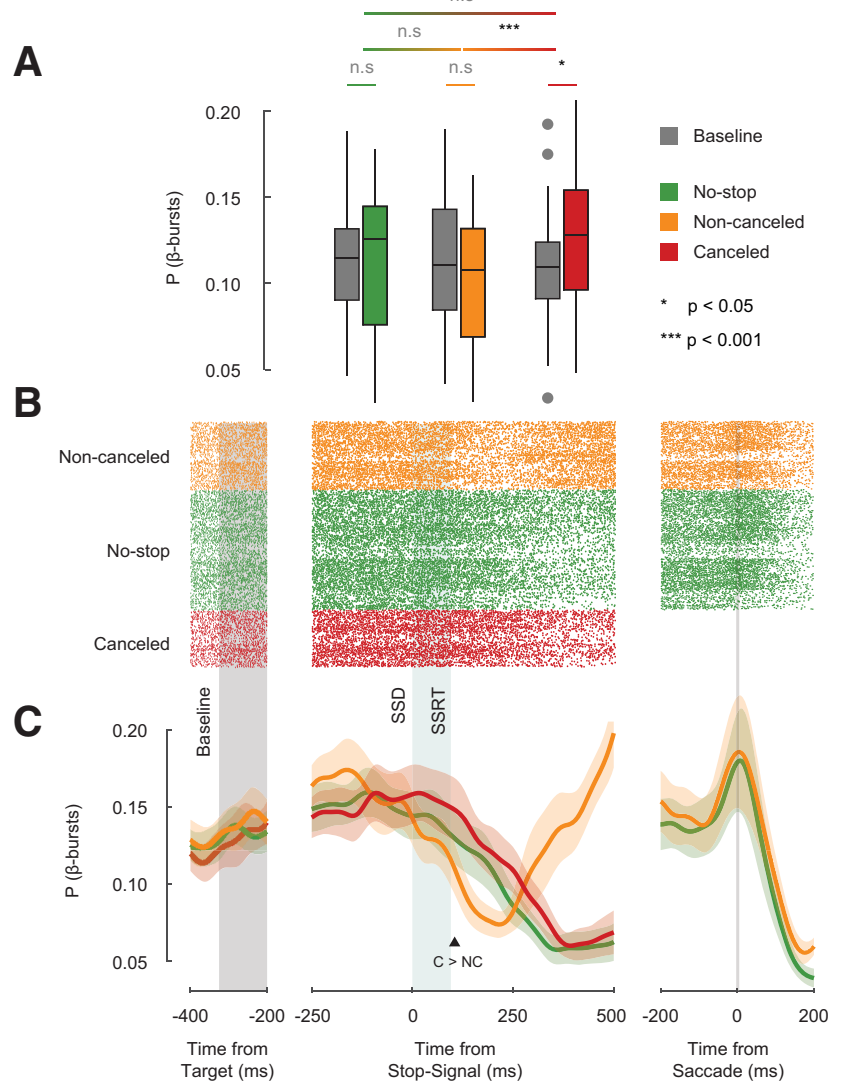

D

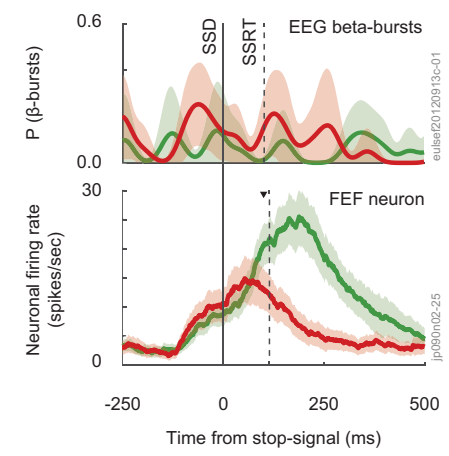

Figure 2. $\beta$-Bursts during stopping. $\boldsymbol{A}$, Boxplots showing the incidence of $\beta$-bursts observed during the STOP process interval (from scheduled SSD to SSRT) during no-stop (green), non-canceled (orange), canceled (red), and during an equivalent period of time before target presentation (gray) . $\beta$-Bursts are observed in $\sim 10-15 \%$ of trials and are slightly but significantly more commonly observed when saccades are inhibited. $\boldsymbol{B}$, Raster plot of $\beta$-bursts aligned on a pretarget baseline interval (left), stop signal (middle), and saccade initiation (right) across all sessions. Each tick-mark shows the time of peak $\beta$-amplitudes satisfying inclusion criteria on each trial. Rasters are shown for non-canceled, no-stop, and canceled trials. The rough equivalence of $\beta$-burst frequency across types of trials is evident, as is the elevation of $\beta$-burst rate at the end of non-canceled error trials. C, $\beta$-Burst density function derived from raster plots. $\beta$-Burst peak times were convolved with a Gaussian function. During the stopping period, $\beta$-bursts were slightly but significantly more common on canceled trials (red line) than on no-stop (green) or non-canceled trials (yellow). $D$, Comparing time course of $\beta$-burst (top) and single neuron discharges (bottom) on canceled and latency-matched no stop signal trials for a single session. At no time did the incidence of $\beta$-bursts on single session differentiate between movement initiation and inhibition. In contrast, as demonstrated previously, the discharge rate of an example FEF movement neuron sampled in one session shows a clear separation between trial types occurring before the STOP process concludes. The neuron was recorded from another monkey in a separate study performing a choice countermanding task (Middlebrooks et al., 2020) and is provided as an example to demonstrate the mechanistic differences between the signals.
Table 2. Percentage of trials (mean \pm SEM) with $\beta$-bursts during a baseline and stopping period, for all trial types

\begin{tabular}{llll}
\hline & No-stop & Non-canceled & Canceled \\
\hline Baseline & $11.6 \pm 0.6 \%$ & $11.7 \pm 0.7 \%$ & $11.2 \pm 0.7 \%$ \\
Stopping period & $11.8 \pm 0.8 \%$ & $10.5 \pm 0.7 \%$ & $13.1 \pm 0.8 \%$ \\
\hline
\end{tabular}

discharge rates of neurons causally involved in movement initiation and inhibition (Middlebrooks et al., 2020; Fig. 2D, bottom panel), $\beta$-burst density functions were so small and noisy that no time distinguished canceled from no stop trials in individual sessions (Fig. $2 D$, upper panel). Notably, across sessions, $\beta$-burst incidence decreases after SSRT in canceled trials. If $\beta$-bursts are supposed to enforce response inhibition, this decay is curious. Because monkeys must sustain fixation for $1500 \mathrm{~ms}$, response inhibition is sustained long after $\beta$-bursts cease. If stopping consists of multiple processes, one responsible for the interruption of the motor plan and another for maintenance of the inhibition goal (Logan and Cowan, 1984; Bompas et al., 2020), perhaps $\beta$-bursts initiate the stop process but do not maintain the inhibition.

The response inhibition function plots the fraction of noncanceled trials in which saccades are produced as a function of stop signal delay (Fig. 1B). The fraction of non-canceled trials is an increasing function of stop signal delay, because movements become less likely to cancel as movement preparation progresses. Using single neuron discharge rates, a neurometric function plots the probability of modulating within SSRT as a function of SSD. The neurometric function derived from the single neuron discharges of movement-related neurons parallels the inhibition function (Brown et al., 2008). Although this relationship has only been demonstrated for neural activity that initiates responses, the relationship with neural activity that instantiate the STOP process is just the inverse with greater likelihood of modulation when the probability of inhibition is highest.

We determined whether a neurometric function derived from $\beta$-bursts parallels the probability of inhibiting a prepared response. For each session, we measured the number of $\beta$-bursts observed on canceled trials at each SSD, $50 \mathrm{~ms}$ before SSRT, using the conventional threshold of $6 \times$ median amplitude plus lower $(2 \times$ median $)$ and higher $(10 \times$ median $)$ thresholds. This $\beta$-burst neurometric function was compared with the probability of inhibiting a response at each SSD (Fig. $3 A$ ) quantitatively through the sum of their squared differences at each stop signal delay. Summed squared differences close to zero indicate similarity of the two measures. Across sessions, the distribution of summed squared differences using the $6 \times$ median threshold was significantly different from zero (one-sample $t$ test: $t_{(28)}=6.70$, $p<0.001, \mathrm{BF}_{10}=56,120.13$; Fig. $3 B$ ). This conclusion did not depend on $\beta$-burst measurement threshold, for even with the lowest threshold finding $\beta$-bursts in only $40 \%$ of canceled trials. No $\beta$-burst measurement threshold produced a neurometric function similar to the inhibition function (one-sample $t$ test at each threshold, $p<10^{-10}$ for all thresholds after corrections for multiple comparisons; Fig. 3B).

\section{$\beta$-Bursts, error monitoring, and executive control}

The stop-signal task is useful for exploring performance monitoring because, by design, errors occur in $50 \%$ of stop-signal trials (here, $40 \%$ of all trials). Using this task, previous work has demonstrated neural activity in supplementary eye field (SEF) that occurs following errors, the magnitude of which is predictive 
of changes in response latencies in the following trial (Stuphorn et al., 2010; Sajad et al., 2019). Hence, we compared the incidence of $\beta$-bursts $100-300$ ms after error and correct saccades, when spiking activity related to errors is maximal. $\beta$-Bursts were significantly more prevalent on error trials $(11.1 \pm 0.7 \%)$ compared with correct trials $(6.7 \pm 0.5 \%)$ during this period (one-way repeated measures ANOVA: $F_{(1,28)}=55.103, p<$ $10^{-3}, \mathrm{BF}_{10}=9068.665$; Fig. $4 A$ ).

Behaviorally, RT on a trial varies according to the outcome of the previous trial (Emeric et al., 2007). Both monkeys produced longer RT in no-stop trials following erroneous non-canceled trials (one-way repeated measures ANOVA with previous trial type as factor, Greenhouse-Geisser corrected, $F_{(1.54,43.30)}=226.341, p<0.001$, $\mathrm{BF}_{10}=6.785 \mathrm{e}+52$, Holm post hoc comparison between no-stop and non-canceled: $\left.p<0.001, \mathrm{BF}_{10}=2.273 \mathrm{e}+7\right)$. To examine whether post-saccade $\beta$-bursts influence post-error slowing, we calculated a post-error slowing index for each session by dividing the mean RT on no-stop trials following non-canceled trials, by the mean RT on no-stop trials following another no-stop trial (Fig. 4B). This value measures the relative change in RT on no-stop trials dependent on the previous trial type. We found that the incidence of $\beta$-bursts after errors bore no relation to RT on the following trial for either monkey (monkey Eu: $R^{2}=0.0333, p=0.571, \mathrm{BF}_{10}=$ 0.521; monkey X: $R^{2}=0.0732, p=0.294, \mathrm{BF}_{10}=0.624$; Fig. $4 C$ ).

Given the outcome-dependent adaption in response latency, we investigated whether the outcome on the previous trial, influenced $\beta$-burst occurrence during the baseline period on the following trial. We found no significant effect of previous trial outcome on the proportion of $\beta$-bursts observed in the baseline of a following no-stop trial (one-way repeated measures ANOVA: $\left.F_{(1.47,41.04)}=3.09, p=0.071, \mathrm{BF}_{10}=1.22\right)$.

\section{Discussion}

We found that the prevalence and timing of the $\beta$-bursts do not account for the likelihood of canceling a planned response. Mirroring findings with human participants, we found macaque monkeys exhibit small but consistent pulses of $\beta$ activity recorded in EEG over medial-frontal cortex during the inhibition of prepared movements (Jana et al., 2020; Wessel, 2020). As previous findings, we observed $\beta$-bursts infrequently ( $\sim 15 \%$ of trials) and not uncommonly in trials in which a response was generated. If $\beta$-bursts cause response inhibition, then they should be more prevalent at earlier SSDs where inhibition is more successful. This relationship between neurometric and psychometric measures of response inhibition has been observed in the discharges of movement-related neurons in frontal eye fields (Brown et al., 2008). However, in the mechanistic, interactive race models of saccade countermanding, unlike the progressive activation of the GO unit over time and across stop signal delays, the action of the STOP unit is effectively all-or-none (Boucher et al., 2007; Logan et al., 2015). Thus, an effective mechanism of response inhibition must happen on every trial in which a response is inhibited. Collectively and unfortunately, these observations raise doubts about the proposal that $\beta$-bursts are a causal mechanism of response inhibition, and limit future applications in devices such as brain-machine interfaces.

Previous findings of $\beta$-bursts over frontal cortex have been interpreted as part of a larger framework proposing that the inferior frontal gyrus of the right hemisphere (rIFG) and the presupplementary motor area (pre-SMA) contribute to reactive control through the hyper-direct pathway (Aron and Poldrack, 2006; Aron et al., 2007). While the rIFG has been implicated in the attentional capture of the stop-signal and initiating the STOP process (Swann et al., 2012; Jana et al., 2020), the role of the preSMA is less clear. Human fMRI studies show greater activity in pre-SMA on stop trials with manual responses (Aron and Poldrack, 2006; Aron et al., 2007; Rae et al., 2015) and in supplementary eye field with saccades (Thakkar et al., 2014). Lesion studies also associate medial frontal areas with impaired stopping of limbs (Floden and Stuss, 2006; Nachev et al., 2007; Sumner et al., 2007) and eyes (Husain et al., 2003). This is mirrored in human electrophysiological evidence reporting stronger signals over pre-SMA during canceled trials (Swann et al., 2012). However, our observation that $\beta$-bursts are slightly more common during response inhibition is unexpected in macaques based on previous neurophysiological results. In single-unit recordings, the modulation of neurons in MFC occurs too late to contribute to the reactive control of movement and instead contributes to performance monitoring and the exertion of proactive control (Emeric et al., 2010; Stuphorn et al., 2010).

Such discrepancies have sparked debate about whether macaques are a useful model of executive control in humans (Cole et al., 2009; Schall and Emeric, 2010). The countermanding task has the advantage for comparisons between macaques and humans because across species it has been tested with the same response modalities, task designs, and measurement scales. Nevertheless, cross-species comparisons require caution because of differences in how the data are collected. First, like previous studies, we cannot localize $\beta$-bursts in scalp data to specific cortical areas, given the spatial resolution of EEG. To draw comparisons with human studies, we have to focused on literature that highlights the role of pre-SMA. These previous studies used tasks 
A
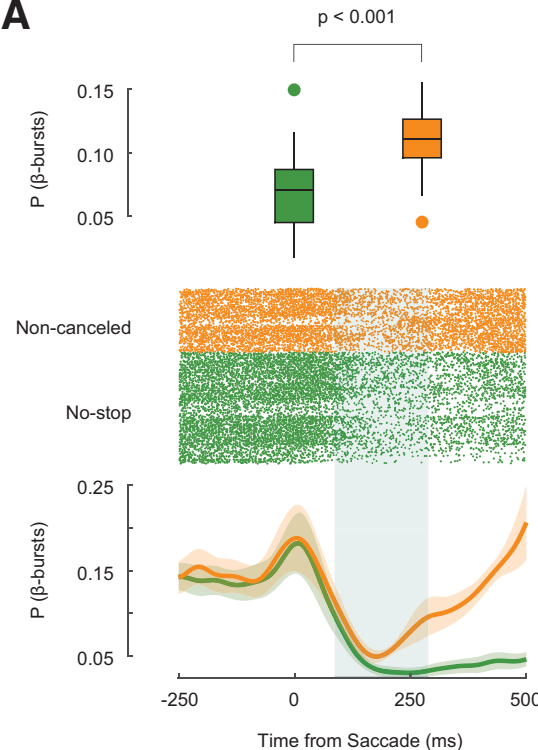

B

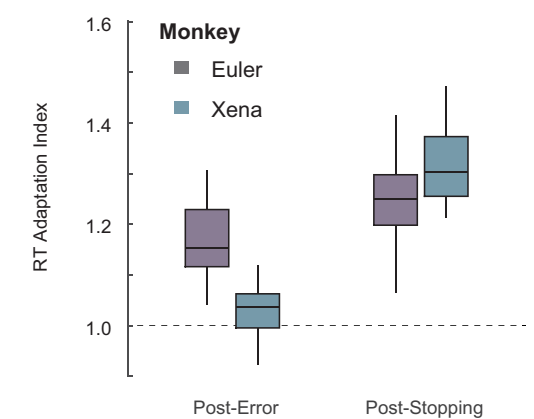

C

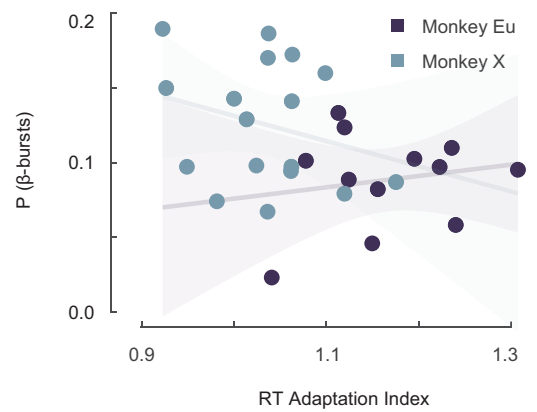

Figure 4. Relationship between $\beta$-bursts and performance monitoring. $A, \beta$-Bursts during error and correct responses. Top, Boxplot showing significantly greater incidence of $\beta$-bursts observed $100-300 \mathrm{~ms}$ following non-canceled (orange) compared with correct saccades (green). Middle, Raster plot aligned on saccade. Each tick-mark shows the time of one $\beta$-burst on a non-canceled (yellow) and no-stop (green) trials. Bottom, $\beta$-Burst density function. Following a saccade, the incidence of bursts on both error and correct trials decreases. This is followed by a pronounced increase in $\beta$-burst frequency $\sim 150 \mathrm{~ms}$ after noncancelled error saccades. $\boldsymbol{B}$, Boxplots of RT adaptation following non-canceled errors and successful cancellations across sessions for Eu (purple) and X (cyan). Values greater than one represent slowing. As observed previously, both monkeys tend to delay responses somewhat after errors and more after successful stopping. $\boldsymbol{C}, \beta$-Bursts are unrelated to RT adaptation. The incidence of $\beta$-bursts observed after errors did not vary as a function of the post-error RT adaptation index across sessions for Eu (purple) or $X$ (cyan). Non-significant regression lines $( \pm 95 \% \mathrm{Cl})$ include 0 slope. which typically required reaching or manual movements. As our study used a saccade-countermanding task, we expect activity to be present in the supplementary eye field, considered to be the "eye area" of the pre-SMA. SEF in macaques is homologous to SEF in humans, as SMA/pre-SMA in macaques is homologous to SMA/pre-SMA in humans (Amiez and Petrides, 2009). In macaques, the pre-SMA is located in the cortex forming the medial wall above cingulate cortex. Thus, from a biophysical standpoint, it is unlikely that the electrical field generated in this area would be captured through the EEG we record. However, SEF is on the dorsomedial convexity and activity in such an area is evident in the overlying EEG (Sajad et al., 2019). Second, compared with experiments with humans, monkeys are well trained and well accustomed to the task, typically completing up to 10 times more trials than humans. This may be demonstrated through our observation that monkeys have a much smaller proportion of trigger failures $(\sim 5 \%)$ compared with what has been previously observed in human $(\sim 20 \%)$ studies (Skippen et al., 2019, 2020). However, although RTs and SSRTs are typically shorter in macaques than humans, the relative relationship between the two is similar between species. Indeed, our first investigation of the stop signal task with macaque monkeys established that their performance matches in various nuanced details that of humans (Hanes and Schall, 1995). Moreover, instantiations of the race model account for both human and macaque countermanding performance equivalently (Boucher et al., 2007; Camalier et al., 2007).

While we found $\beta$-bursts were more common during response inhibition, we also found they were more common following errors, suggesting a contribution to performance monitoring and executive control. This observation is consistent with patterns of spiking activity described in medial frontal cortex (Stuphorn et al., 2000; Sajad et al., 2019) and complementing previous observations that error, conflict, and reward monitoring are typically associated with $\theta$ (4$8 \mathrm{~Hz}$ ) band modulation (Luu et al., 2004; Trujillo and Allen, 2007; Cohen et al., 2008; Cavanagh et al., 2009; Nigbur et al., 2011; Amarante et al., 2017). Over medial frontal cortex, $\beta$ power is elevated when cognitive control was required and following negative feedback (Stoll et al., 2016). Specifically, in a change-stop-signal task, previous work has highlighted greater $\beta$ activity on canceled compared with non-canceled trials in pre-SMA, following the completion of the STOP process (Jha et al., 2015). This has also been demonstrated in a combined Flanker stop-signal task, in which $\beta$ activity increased following the commission of an error (MarcoPallarés et al., 2008; Beyer et al., 2012). Interestingly, the magnitude of this $\beta$ activity correlated with the $\theta$-activity underlying the ERN (Marco-Pallarés et al., 2008).

While it has been previous argued that there is mixed evidence for a relationship between the error-related negativity and performance adjustments (Gehring et al., 1993; Gehring and Fencsik, 2001; Rodriguez-Fornells et al., 2002; Hajcak et al., 2003; Kerns et al., 2004; Holroyd et al., 2005; Ladouceur et al., 2007; West and Travers, 2008; Núñez Castellar et al., 2010; Godlove et al., 2011b; Reinhart et al., 2012; Fu et al., 2019), recent meta-analyses and large-scale studies have demonstrated a more robust relationship between single-trial ERN amplitude and post-error slowing. In a meta-analysis of mid-frontal $\theta$, Cavanagh and Shackman (2015) showed systematic evidence that increases in the magnitude of the ERN were coupled with greater post-error slowing; the magnitude of this effect was over twice as large within-subjects 
compared with between-subjects. Supporting this, a recent large-scale study of $\sim 900$ participants demonstrated that greater ERN amplitude was associated with greater posterror slowing within subjects, but not between (Fischer et al., 2016). In contrast to previous findings, we found incidence of $\beta$-bursts after errors did not predict post-error adjustments in RT. Previous observations have reported increased $\beta$ activity in pre-SMA was associated with greater post-error slowing (Marco-Pallarés et al., 2008; Jha et al., 2015) but not with the latency of corrective responses (Marco-Pallarés et al., 2008).

Although we have observed these significant changes in $\beta$-burst activity during response inhibition and executive control, $\beta$-bursts were neither necessary or sufficient for stopping and were also commonly observed on trials with erroneous saccades executed. Furthermore, changes in stopping performance were not reflected through changes in the proportion of $\beta$-bursts observed. The overall low prevalence of $\beta$-bursts must be noted. This resulted in $\beta$-burst density functions which were small and noisy compared with single neuron spike density functions. Given that $\beta$-bursts are relatively uncommon and non-specific, we draw inferences only cautiously. Several alternative hypotheses can be considered. First, medial frontal $\beta$-bursts may not be specific to stopping. Indeed, we observed pronounced changes in $\beta$-burst occurrence after errors. Second, as argued previously (Jana et al., 2020; Wessel, 2020), EEG has a poor signal-to-noise ratio. This issue can be addressed in future work by measuring the occurrence of $\beta$-bursts in intracranial signals recorded in medial frontal areas. Finally, methods for detecting $\beta$-bursts could miss significant but subthreshold bursts. Perhaps, more sensitive methods can be developed.

To conclude, by replicating measurements of $\beta$-bursts in EEG of macaque monkeys, we establish an animal model of this phenomenon. First, we demonstrated $\beta$-bursts were more prevalent when movements were successfully inhibited. Second, we demonstrated a greater incidence of $\beta$-bursts over the medial frontal cortex when a movement was erroneously executed. However, in neither context were $\beta$-bursts frequent enough to account for behavior. Given the pulses of $\beta$-bursts at different stages during the task, it is uncertain whether they index different mechanisms or are produced by one mechanism at different times. In previous work, this uncertainty and apparent absence of causal efficacy has been explained as a consequence of poor signal-to-noise ratio of noninvasive EEG recordings. Establishing the same phenomena in a monkey model engenders confidence in proceeding with the systematic investigation of the neural mechanisms of $\beta$-burst generation in the cerebral cortex during countermanding or other tasks. Future studies which sample $\beta$-bursts across the layers of the cortex can provide insights into the mechanisms of their generation and possibly help elucidate the role of $\beta$-bursts during response inhibition and executive control.

\section{References}

Amarante LM, Caetano MS, Laubach M (2017) Medial frontal theta is entrained to rewarded actions. J Neurosci 37:10757-10769.

Amiez C, Petrides M (2009) Anatomical organization of the eye fields in the human and non-human primate frontal cortex. Prog Neurobiol 89:220230.

Aron AR, Poldrack RA (2006) Cortical and subcortical contributions to Stop signal response inhibition: role of the subthalamic nucleus. J Neurosci 26:2424-2433
Aron AR, Behrens TE, Smith S, Frank MJ, Poldrack RA (2007) Triangulating a cognitive control network using diffusion-weighted magnetic resonance imaging (MRI) and functional MRI. J Neurosci 27:3743-3752.

Beyer F, Münte TF, Fischer J, Krämer UM (2012) Neural aftereffects of errors in a stop-signal task. Neuropsychologia 50:3304-3312.

Bompas A, Campbell AE, Sumner P (2020) Cognitive control and automatic interference in mind and brain: a unified model of saccadic inhibition and countermanding. Psychol Rev 127:524-561.

Boucher L, Palmeri TJ, Logan GD, Schall JD (2007) Inhibitory control in mind and brain: an interactive race model of countermanding saccades. Psychol Rev 114:376-397.

Brockett AT, Hricz NW, Tennyson SS, Bryden DW, Roesch MR (2020) Neural signals in red nucleus during reactive and proactive adjustments in behavior. J Neurosci 40:4715-4726.

Brown JW, Hanes DP, Schall JD, Stuphorn V (2008) Relation of frontal eye field activity to saccade initiation during a countermanding task. Exp Brain Res 190:135-151.

Cabel DW, Armstrong IT, Reingold E, Munoz DP (2000) Control of saccade initiation in a countermanding task using visual and auditory stop signals. Exp Brain Res 133:431-441.

Camalier CR, Gotler A, Murthy A, Thompson KG, Logan GD, Palmeri TJ, Schall JD (2007) Dynamics of saccade target selection: race model analysis of double step and search step saccade production in human and macaque. Vision Res 47:2187-2211.

Cavanagh JF, Shackman AJ (2015) Frontal midline theta reflects anxiety and cognitive control: meta-analytic evidence. J Physiol Paris 109:3-15.

Cavanagh JF, Cohen MX, Allen JJ (2009) Prelude to and resolution of an error: EEG phase synchrony reveals cognitive control dynamics during action monitoring. J Neurosci 29:98-105.

Cohen MX, Ridderinkhof KR, Haupt S, Elger CE, Fell J (2008) Medial frontal cortex and response conflict: evidence from human intracranial EEG and medial frontal cortex lesion. Brain Res 1238:127-142.

Cole MW, Yeung N, Freiwald WA, Botvinick M (2009) Cingulate cortex: diverging data from humans and monkeys. Trends Neurosci 32:566574.

Colonius H, Özyurt J, Arndt PA (2001) Countermanding saccades with auditory stop signals: testing the race model. Vision Res 41:19511968.

De Jong R, Coles MG, Logan GD (1995) Strategies and mechanisms in nonselective and selective inhibitory motor control. J Exp Psychol Hum Percept Perform 21:498-511.

Emeric EE, Brown JW, Boucher L, Carpenter RH, Hanes DP, Harris R, Logan GD, Mashru RN, Paré M, Pouget P, Stuphorn V, Taylor TL, Schall JD (2007) Influence of history on saccade countermanding performance in humans and macaque monkeys. Vision Res 47:35-49.

Emeric EE, Leslie M, Pouget P, Schall JD (2010) Performance monitoring local field potentials in the medial frontal cortex of primates: supplementary eye field. J Neurophysiol 104:1523-1537.

Fischer AG, Danielmeier C, Villringer A, Klein TA, Ullsperger M (2016) Gender influences on brain responses to errors and post-error adjustments. Sci Rep 6:24435.

Floden D, Stuss DT (2006) Inhibitory control is slowed in patients with right superior medial frontal damage. J Cogn Neurosci 18:1843-1849.

Fu Z, Wu DJ, Ross I, Chung JM, Mamelak AN, Adolphs R, Rutishauser U (2019) Single-neuron correlates of error monitoring and posterror adjustments in human medial frontal cortex. Neuron 101:165177.e5.

Gehring WJ, Fencsik DE (2001) Functions of the medial frontal cortex in the processing of conflict and errors. J Neurosci 21:9430-9437.

Gehring WJ, Goss B, Coles MG, Meyer DE, Donchin E (1993) A neural system for error detection and compensation. Psychol Sci 4:385390.

Godlove DC, Schall JD (2016) Microsaccade production during saccade cancelation in a stop-signal task. Vision Res 118:5-16.

Godlove DC, Garr AK, Woodman GF, Schall JD (2011a) Measurement of the extraocular spike potential during saccade countermanding. J Neurophysiol 106:104-114.

Godlove DC, Emeric EE, Segovis CM, Young MS, Schall JD, Woodman GF (2011b) Event-related potentials elicited by errors during the stop-signal task. I. Macaque monkeys. J Neurosci 31:15640-15649. 
Godlove DC, Maier A, Woodman GF, Schall JD (2014) Microcircuitry of agranular frontal cortex: testing the generality of the canonical cortical microcircuit. J Neurosci 34:5355-5369.

Hajcak G, McDonald N, Simons RF (2003) To err is autonomic: error-related brain potentials, ANS activity, and post-error compensatory behavior. Psychophysiology 40:895-903.

Hanes DP, Schall JD (1995) Countermanding saccades in macaque. Vis Neurosci 12:929-937.

Hanes DP, Carpenter RH (1999) Countermanding saccades in humans. Vision Res 39:2777-2791.

Hanes DP, Patterson WF 2nd, Schall JD (1998) Role of frontal eye fields in countermanding saccades: visual, movement, and fixation activity. J Neurophysiol 79:817-834.

Holroyd CB, Yeung N, Coles MG, Cohen JD (2005) A mechanism for error detection in speeded response time tasks. J Exp Psychol Gen 134:163191.

Husain M, Parton A, Hodgson TL, Mort D, Rees G (2003) Self-control during response conflict by human supplementary eye field. Nat Neurosci 6:117-118.

Jana S, Hannah R, Muralidharan V, Aron AR (2020) Temporal cascade of frontal, motor and muscle processes underlying human action-stopping. Elife 9.

Jha A, Nachev P, Barnes G, Husain M, Brown P, Litvak V (2015) The frontal control of stopping. Cereb Cortex 25:4392-4406.

Kawagoe R, Takikawa Y, Hikosaka O (1998) Expectation of reward modulates cognitive signals in the basal ganglia. Nat Neurosci 1:411-416.

Kerns JG, Cohen JD, MacDonald AW 3rd, Cho RY, Stenger VA, Carter CS (2004) Anterior cingulate conflict monitoring and adjustments in control. Science 303:1023-1026.

Kok A, Ramautar JR, De Ruiter MB, Band GP, Ridderinkhof KR (2004) ERP components associated with successful and unsuccessful stopping in a stop-signal task. Psychophysiology 41:9-20.

Kornylo K, Dill N, Saenz M, Krauzlis RJ (2003) Cancelling of pursuit and saccadic eye movements in humans and monkeys. J Neurophysiol 89:29842999.

Ladouceur CD, Dahl RE, Carter CS (2007) Development of action monitoring through adolescence into adulthood: ERP and source localization. Dev Sci 10:874-891.

Logan GD, Cowan WB (1984) On the ability to inhibit thought and action a theory of an act of control. Psychol Rev 91:295-327.

Logan GD, Yamaguchi M, Schall JD, Palmeri TJ (2015) Inhibitory control in mind and brain 2.0: blocked-input models of saccadic countermanding. Psychol Rev 122:115-147.

Luu P, Tucker DM, Makeig S (2004) Frontal midline theta and the errorrelated negativity: neurophysiological mechanisms of action regulation. Clin Neurophysiol 115:1821-1835.

Marco-Pallarés J, Camara E, Münte TF, Rodríguez-Fornells A (2008) Neural mechanisms underlying adaptive actions after slips. J Cogn Neurosci 20:1595-1610

Matzke D, Love J, Heathcote A (2017) A Bayesian approach for estimating the probability of trigger failures in the stop-signal paradigm. Behav Res Methods 49:267-281

Matzke D, Dolan CV, Logan GD, Brown SD, Wagenmakers EJ (2013a) Bayesian parametric estimation of stop-signal reaction time distributions. J Exp Psychol Gen 142:1047-1073.

Matzke D, Love J, Wiecki TV, Brown SD, Logan GD, Wagenmakers EJ (2013b) Release the BEESTS: Bayesian estimation of ex-Gaussian STopsignal reaction time distributions. Front Psychol 4:918.

Middlebrooks PG, Zandbelt BB, Logan GD, Palmeri TJ, Schall JD (2020) Countermanding perceptual decision-making. iScience 23:100777.

Mirabella G, Pani P, Ferraina S (2011) Neural correlates of cognitive control of reaching movements in the dorsal premotor cortex of rhesus monkeys. J Neurophysiol 106:1454-1466.

Morein-Zamir S, Kingstone A (2006) Fixation offset and stop signal intensity effects on saccadic countermanding: a crossmodal investigation. Exp Brain Res 175:453-462.

Murthy A, Ray S, Shorter SM, Schall JD, Thompson KG (2009) Neural control of visual search by frontal eye field: effects of unexpected target displacement on visual selection and saccade preparation. J Neurophysiol 101:2485-2506.
Nachev P, Wydell H, O'Neill K, Husain M, Kennard C (2007) The role of the pre-supplementary motor area in the control of action. Neuroimage 36: T155-T163.

Nelson MJ, Boucher L, Logan GD, Palmeri TJ, Schall JD (2010) Nonindependent and nonstationary response times in stopping and stepping saccade tasks. Atten Percept Psychophys 72:1913-1929.

Nigbur R, Ivanova G, Stürmer B (2011) Theta power as a marker for cognitive interference. Clin Neurophysiol 122:2185-2194.

Núñez Castellar E, Kühn S, Fias W, Notebaert W (2010) Outcome expectancy and not accuracy determines posterror slowing: ERP support. Cogn Affect Behav Neurosci 10:270-278.

Paré M, Hanes DP (2003) Controlled movement processing: superior colliculus activity associated with countermanded saccades. J Neurosci 23:64806489.

Rae CL, Hughes LE, Anderson MC, Rowe JB (2015) The prefrontal cortex achieves inhibitory control by facilitating subcortical motor pathway connectivity. J Neurosci 35:786-794.

Reinhart RM, Carlisle NB, Kang MS, Woodman GF (2012) Eventrelated potentials elicited by errors during the stop-signal task. II: Human effector-specific error responses. J Neurophysiol 107:27942807.

Rodriguez-Fornells A, Kurzbuch AR, Münte TF (2002) Time course of error detection and correction in humans: neurophysiological evidence. J Neurosci 22:9990-9996.

Sajad A, Godlove DC, Schall JD (2019) Cortical microcircuitry of performance monitoring. Nat Neurosci 22:265-274.

Scangos KW, Stuphorn V (2010) Medial frontal cortex motivates but does not control movement initiation in the countermanding task. J Neurosci 30:1968-1982.

Schall JD, Emeric EE (2010) Conflict in cingulate cortex function between humans and macaque monkeys: more apparent than real. Brain Behav Evol 75:237-238.

Schmidt R, Leventhal DK, Mallet N, Chen F, Berke JD (2013) Canceling actions involves a race between basal ganglia pathways. Nat Neurosci $16: 1118-1124$

Shin H, Law R, Tsutsui S, Moore CI, Jones SR (2017) The rate of transient beta frequency events predicts behavior across tasks and species. Elife 6

Skippen P, Matzke D, Heathcote A, Fulham WR, Michie P, Karayanidis F (2019) Reliability of triggering inhibitory process is a better predictor of impulsivity than SSRT. Acta Psychol (Amst) 192:104-117.

Skippen P, Fulham WR, Michie PT, Matzke D, Heathcote A, Karayanidis F (2020) Reconsidering electrophysiological markers of response inhibition in light of trigger failures in the stop-signal task. Psychophysiology e13619.

Stahl J, Gibbons H (2007) Dynamics of response-conflict monitoring and individual differences in response control and behavioral control: an electrophysiological investigation using a stop-signal task. Clin Neurophysiol 118:581-596.

Stoll FM, Wilson CRE, Faraut MCM, Vezoli J, Knoblauch K, Procyk E (2016) The effects of cognitive control and time on frontal beta oscillations. Cereb Cortex 26:1715-1732.

Stuphorn V, Taylor TL, Schall JD (2000) Performance monitoring by the supplementary eye field. Nature 408:857-860.

Stuphorn V, Brown JW, Schall JD (2010) Role of supplementary eye field in saccade initiation: executive, not direct, control. J Neurophysiol 103:801816.

Sumner P, Nachev P, Morris P, Peters AM, Jackson SR, Kennard C, Husain M (2007) Human medial frontal cortex mediates unconscious inhibition of voluntary action. Neuron 54:697-711.

Swann NC, Cai W, Conner CR, Pieters TA, Claffey MP, George JS, Aron AR, Tandon N (2012) Roles for the pre-supplementary motor area and the right inferior frontal gyrus in stopping action: electrophysiological responses and functional and structural connectivity. Neuroimage 59:2860-2870

Thakkar KN, Schall JD, Boucher L, Logan GD, Park S (2011) Response inhibition and response monitoring in a saccadic countermanding task in schizophrenia. Biol Psychiatry 69:55-62.

Thakkar KN, van den Heiligenberg FM, Kahn RS, Neggers SF (2014) Frontal-subcortical circuits involved in reactive control and monitoring of gaze. J Neurosci 34:8918-8929. 
Thakkar KN, Schall JD, Logan GD, Park S (2015) Cognitive control of gaze in bipolar disorder and schizophrenia. Psychiatry Res 225:254-262.

Trujillo LT, Allen JJ (2007) Theta EEG dynamics of the error-related negativity. Clin Neurophysiol 118:645-668.

Verbruggen F, Logan GD (2008) Response inhibition in the stop-signal paradigm. Trends Cogn Sci 12:418-424.

Verbruggen F, Aron AR, Band GP, Beste C, Bissett PG, Brockett AT, Brown JW, Chamberlain SR, Chambers CD, Colonius H, Colzato LS, Corneil BD, Coxon JP, Dupuis A, Eagle DM, Garavan H, Greenhouse I, Heathcote A, Huster RJ, Jahfari S, et al. (2019) A consensus guide to capturing the ability to inhibit actions and impulsive behaviors in the stopsignal task. Elife 8:e46323.

Walton MM, Gandhi NJ (2006) Behavioral evaluation of movement cancellation. J Neurophysiol 96:2011-2024.
Wattiez N, Poitou T, Rivaud-Péchoux S, Pouget P (2016) Evidence for spatial tuning of movement inhibition. Exp Brain Res 234:19571966.

Wessel JR (2020) $\beta$-Bursts reveal the trial-to-trial dynamics of movement initiation and cancellation. J Neurosci 40:411-423.

Wessel JR, Aron AR (2015) It's not too late: the onset of the frontocentral P3 indexes successful response inhibition in the stop-signal paradigm. Psychophysiol 52:472-480.

West R, Travers S (2008) Tracking the temporal dynamics of updating cognitive control: an examination of error processing. Cereb Cortex 18:11121124.

Woodman GF (2012) Homologues of human ERP components in nonhuman primates. In: Oxford handbook of event-related potential components, Ed 1, pp 611-626. New York: Oxford University Press. 